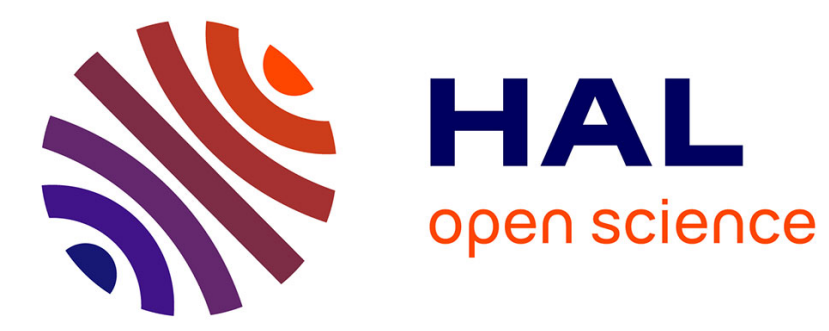

\title{
Cooperative Aerial Load Transportation via Sampled Communication
}

Enrica Rossi, Marco Tognon, Ruggero Carli, Luca Schenato, Juan Cortés, Antonio Franchi

\section{To cite this version:}

Enrica Rossi, Marco Tognon, Ruggero Carli, Luca Schenato, Juan Cortés, et al.. Cooperative Aerial Load Transportation via Sampled Communication. IEEE Control Systems Letters, 2020, 4 (2), pp.277282. 10.1109/LCSYS.2019.2924413 . hal-02170909

\section{HAL Id: hal-02170909 \\ https://hal.laas.fr/hal-02170909}

Submitted on 2 Jul 2019

HAL is a multi-disciplinary open access archive for the deposit and dissemination of scientific research documents, whether they are published or not. The documents may come from teaching and research institutions in France or abroad, or from public or private research centers.
L'archive ouverte pluridisciplinaire HAL, est destinée au dépôt et à la diffusion de documents scientifiques de niveau recherche, publiés ou non, émanant des établissements d'enseignement et de recherche français ou étrangers, des laboratoires publics ou privés. 


\title{
Cooperative Aerial Load Transportation via Sampled Communication
}

\author{
Enrica Rossi ${ }^{1}$, Marco Tognon ${ }^{2}$, Ruggero Carli ${ }^{1}$, Luca Schenato ${ }^{1}$, Juan Cortés ${ }^{2}$, Antonio Franchi ${ }^{2}$,
}

\begin{abstract}
In this work, we propose a feedback-based motion planner for a class of multi-agent manipulation systems with a sparse kinematics structure. In other words, the agents are coupled together only by the transported object. The goal is to steer the load into a desired configuration. We suppose that a global motion planner generates a sequence of desired configurations that satisfy constraints as obstacles and singularities avoidance. Then, a local planner receives these references and generates the desired agents velocities, which are converted into force inputs for the vehicles. We focus on the local planner design both in the case of continuously available measurements and when they are transmitted to the agents via sampled communication. For the latter problem, we propose two strategies. The first is the discretization of the continuous-time strategy that preserves stability and guarantees exponential convergence regardless of the sampling period. In this case, the planner gain is static and computed off-line. The second strategy requires to collect the measurements from all sensors and to solve online a set of differential equations at each sampling period. However, it has the advantage to provide doubly exponential convergence. Numerical simulations of these strategies are provided for the cooperative aerial manipulation of a cable-suspended load.
\end{abstract}

\section{INTRODUCTION}

Unamnned Aerial Vehicles (UAVs) have received a lot of attention in the last decade thanks to their broad field of applications. In fact, they are employed to perform diverse complex tasks from search and rescue to load manipulation and transportation [1], [2]. Due to the limited payload of commercially available vehicles, a group of robots is rather employed to transport and manipulate payloads, e.g., for construction and assembly tasks. The most direct approach to control such a multi-robot system is by a centralized kinematic/dynamic inversion [3]-[5]. However, a distributed approach, where the communication among agents is restricted to neighbors [6], [7] or not employed at all [8], [9], is more favorable. This technique guarantees major robustness and flexibility w.r.t. a centralized one, where a central unit handles all the computational load and if the latter fails, then the whole task fails. However, the distributed approach lacks global information as the load state and parameters, or the total number of robots. This aspect increases the difficulty of the controller design and might degrade the performance as well. Moreover, many communication-less approaches rely

This work Partially funded by: EU Horizon 2020 grant agreement No 644271 AEROARMS, and the ANR, Project ANR-17- CE33-0007 MuRoPhen and by University of Padova grant "Magic" SCHE_SID17_01.

${ }^{1}$ Department of Information Engineering, University of Padova, Italy enrica.rossi.1@studenti.unipd.it, carlirugedei.unipd.it, schenato@dei. unipd.it

${ }^{2}$ LAAS-CNRS, Université de Toulouse, CNRS, Toulouse, France, marco.tognon@laas.fr, juan.cortes@laas.fr, antonio.franchi@laas.fr on a leader-follower paradigm: the followers track the leader who is the only agent aware of the desired task. Hence i) if the leader fails, then the task will fail too, and ii) if one follower fails, the formation might be loosen. In this scenario, the communication is done implicitly by the forces exchanged through the load [10], [11]. However, if a very precise tracking is required, the force feedback is not enough and a pose feedback from the load is needed. This can be measured by a sensor placed on the load, or retrieved from the robots pose if those are rigidly connected to the load. Since in this work the load is suspended by cables, only the first option is feasible. In particular, the load sensory suit shall send the estimated load pose to the robots via a communication channel.

To the best of our knowledge, in this work we investigate for the first time the problem of cooperative aerial load transportation via sampled communication. In fact, the bandwidth required to fulfill real-time requirements in robotic manipulation is larger w.r.t. the one provided by today's WiFi. Therefore, the frequency of communication is not high and neither constant. We tackle these problems by considering an architecture where a global motion planner [5] generates off-line a sequence of reference points, taking into account obstacles and singularities avoidance. These points are then loaded in the computational unit, that in our scenario can be placed on the transported platform or on the vehicles, depending on the strategy. Then, to connect each pair of consecutive points along the path computed off-line, we propose a method that computes online the reference vehicles velocities, guaranteeing the stability and the exponential convergence of the pose load error, even when the communication is not continuous. In the following, we call this method a local motion planner. The design of this planner is our main contribution: we firstly define it in the continuous time scenario and later extend the analysis to the more realistic case in which the measured variables are transmitted via sampled communication to the low-level agent controllers. Note that we focus only on the kinematic model since it is assumed that each agent is endowed with a dynamic model-based local controller. For this problem, we propose two strategies: a static feedback technique that exploits the sparsity of the system model and an adaptive one, where the planner gain is adapted according to the system state. The second strategy provides faster convergence rate w.r.t. the first one, but cannot be implemented in a distributed way.

The paper is organized as follows: in Sec.II we formalize the problem for a particular class of systems. In Sec.III we analyze the continuous-time scenario, extended to the 


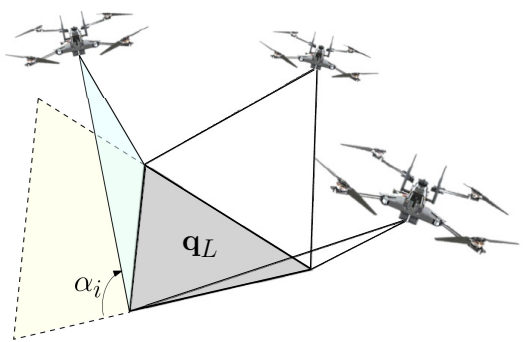

Fig. 1: Example of a multi-agent system that transports a payload.

discrete-time case in Sec.IV. In Sec.V numerical simulations are described.

\section{MODELING AND PROBLEM FORMULATION}

\section{A. Kinematics of multi-agent systems}

In this section we describe the kinematic model of a multiagent system composed by $N$ actuated agents interacting through a common object $L$ (the load to be manipulated) by means of passive mechanical connections, such as cables, bars, joints, etc.. Agents can be, e.g., 1-DoF actuated cables of variable length or even 3-DoF actuated points flying in the 3D space. The kinematic model is derived as in [5]:

$$
\left[\begin{array}{lll}
\mathbf{p}_{1} & \ldots & \mathbf{p}_{N}
\end{array}\right]^{\top}=\mathbf{h}(\mathbf{q})=\left[\mathbf{h}^{(1)}\left(\mathbf{q}_{1}, \mathbf{q}_{L}\right) \ldots \mathbf{h}^{(N)}\left(\mathbf{q}_{N}, \mathbf{q}_{L}\right)\right]^{\top}
$$

This function maps the Lagrangian coordinates of the system $\mathbf{q}=\left[\begin{array}{lll}\mathbf{q}_{1}^{\top} \cdots \mathbf{q}_{N}^{\top} & \mathbf{q}_{L}^{\top}\end{array}\right]^{\top} \in \mathbb{R}^{m}$ to the vector collecting the agents configurations $\mathbf{p}=\left[\begin{array}{lll}\mathbf{p}_{1} & \ldots & \mathbf{p}_{N}\end{array}\right]^{\top} \in \mathbb{R}^{n}$ where $\mathbf{p}_{i} \in \mathbb{R}^{n_{i}}$ represents the cables lengths in a cable-driven robot or the positions of the flying points in an aerial system. In particular, $\mathbf{q}_{i} \in \mathbb{R}^{m_{i}}$ gathers the angles between the common object $L$ and the $i$-th robot, and $\mathbf{q}_{L} \in \mathbb{R}^{m_{L}}$ represents the pose (position and orientation) of the manipulated object itself. Note that $m_{i}=0$ if $\mathbf{p}_{i}$ can be fully described by the only load pose $\mathbf{q}_{L}$. Otherwise, if $\mathbf{p}_{i}$ depends also on $\mathbf{q}_{i}$, then $m_{i}=1$ if one angle is sufficient to express the positions $\mathbf{p}_{i}$ w.r.t. the load pose (as in Fig.1); if a single cable connects a robot to the load, then the cable orientation is described by two angles, i.e. $m_{i}=2$. Note that we do not consider rotations of the cables about their own axis, hence $m_{i} \leq 2$. A key feature of the systems considered in this work is that they exhibit a star-like interaction topology where each agent is connected to a central unit (the load). This topology is more evident when considering its differential kinematics:

$$
\dot{\mathbf{p}}=\mathbf{A}_{\mathbf{q}} \dot{\mathbf{q}}
$$

where the Jacobian $\mathbf{A}_{\mathbf{q}}=\frac{\partial \mathbf{h}(\mathbf{q})}{\partial \mathbf{q}} \in \mathbb{R}^{n \times m}$ has the structure

$$
\mathbf{A}_{\mathbf{q}}=\left[\begin{array}{ccc|c}
\mathbf{A}_{\mathbf{q}_{1}}^{(1)} & & \mathbf{0} & \mathbf{A}_{\mathbf{q}_{L}}^{(1)} \\
& \ddots & & \vdots \\
\mathbf{0} & & \mathbf{A}_{\mathbf{q}_{N}}^{(N)} & \mathbf{A}_{\mathbf{q}_{L}}^{(N)}
\end{array}\right],
$$

and $\mathbf{A}_{\mathbf{q}_{i}}^{(i)}=\frac{\partial \mathbf{h}^{i}(\mathbf{q})}{\partial \mathbf{q}_{i}} \in \mathbb{R}^{n_{i} \times m_{i}}$ and $\mathbf{A}_{\mathbf{q}_{L}}^{(i)}=\frac{\partial \mathbf{h}^{i}(\mathbf{q})}{\partial \mathbf{q}_{L}} \in \mathbb{R}^{n_{i} \times m_{L}}$.

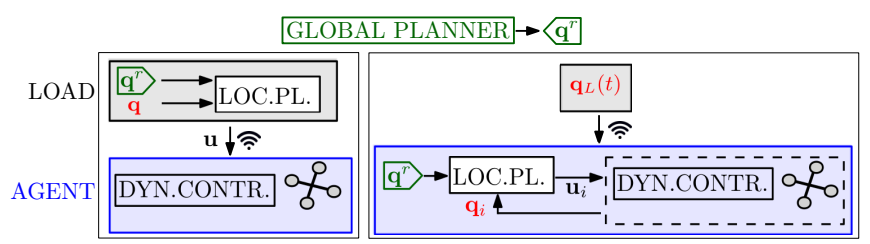

Fig. 2: Representation of (5) (left) and (6) (right). The load is represented in gray, each agent (dynamical controller plus robot) in blue, the measurements from the sensors in red and the global planner in green. The dynamical controller converts $\mathbf{u}$ into forces for the robot. The wireless symbol refers to sampled communication.

\section{B. Problem Formulation}

The goal of the paper is to design a feedback-based local planner in order to steer the system from an initial configuration $\mathbf{q}^{0}$ to a desired one $\mathbf{q}^{r}$. In general, $\mathbf{q}^{r}$ could be a waypoint sampled at the time instant $t_{\ell}, \ell \in \mathbb{N}$ from a trajectory $\mathbf{q}^{p}(t)$, with $t \in \mathbb{R}$, generated off-line by a global path planner. This can take into account high-level performance metrics such as obstacles avoidance, minimum energy trajectories and singularity avoidance (i.e., the configurations s.t. the Jacobian $\mathbf{A}_{\mathbf{q}^{p}(t)}$ is not full rank). The objective is to steer the system from a configuration $\mathbf{q}^{0} \approx \mathbf{q}^{p}\left(t_{\ell}\right)$ to a final one $\mathbf{q}^{r} \approx \mathbf{q}^{p}\left(t_{\ell+1}\right)$ faster than the intersample interval $\Delta t=t_{\ell+1}-t_{\ell}$. We assume that each agent has an inner-loop control sufficiently fast with respect to the dynamics of the entire system, such that the velocities of the variable vector $\mathbf{p}$ are fully controllable, i.e.:

$$
\dot{\mathbf{p}}(t)=\mathbf{u}(t),
$$

where $\mathbf{u}=\left[\mathbf{u}_{1}^{\top} \cdots \mathbf{u}_{N}^{\top}\right]^{\top} \in \mathbb{R}^{n}$. We consider two possible architectures, depicted in Fig.2:

$$
\begin{aligned}
& \mathbf{u}_{i}(t)=\kappa_{i}^{s}\left(\mathbf{q}_{i}(t), \mathbf{q}_{L}(t) ; \mathbf{q}_{i}^{r}, \mathbf{q}_{L}^{r}\right), \quad i=1, \ldots, N \\
& \mathbf{u}_{i}(t)=\kappa_{i}^{c}\left(\mathbf{q}(t) ; \mathbf{q}^{r}\right), \quad i=1, \ldots, N
\end{aligned}
$$

where the former tries to maintain the same sparsity of the Jacobian while the latter exploits the full knowledge of ${ }^{1} \mathbf{q}$. We will show that if $\mathbf{q}(t)$ is continuously accessible to the local planner, then (5) is sufficient to drive the system from $\mathbf{q}^{0}$ to $\mathbf{q}^{r}$ very effectively. On the other hand, under the more realistic scenario when some of the components of $\mathbf{q}(t)$ need to be sampled and transmitted via wireless, then the two architectures give rise to two different strategies with different performance and computational requirements. Before proceeding, we define the set $\mathscr{B}_{r}\left(\mathbf{q}^{r}\right):=\left\{\mathbf{q} \in \mathbb{R}^{m} \mid\left\|\mathbf{q}-\mathbf{q}^{r}\right\|<\right.$ $r, r>0\}$, where $\|\cdot\|$ indicates the Euclidean norm and the following assumption:

\section{Assumption 1. The following relations hold:}

1) $n=m$, i.e., $\mathbf{A}_{\mathbf{q}} \in \mathbb{R}^{n \times n}$

2) the matrix $\mathbf{A}_{\mathbf{q}}$ is invertible and the map $\mathbf{h}$ is twice continuously differentiable for all $q \in \mathscr{B}_{r}\left(\mathbf{q}^{r}\right)$. In addition, these properties can be extended by continuity on the closure of such set, defined as $\overline{\mathscr{B}}_{r}\left(\mathbf{q}^{r}\right)$.

\footnotetext{
${ }^{1}$ Note that we do not pursue control strategies of type $\mathbf{u}(t)=\kappa\left(\mathbf{p}(t) ; \mathbf{p}^{r}\right.$ where $\mathbf{p}^{r}:=\mathbf{h}\left(\mathbf{q}^{\mathbf{r}}\right)$ since the function $\mathbf{h}$ might not be perfectly known and avoidance of singular configurations cannot be guaranteed.
} 


\section{3) $\mathbf{q}^{0} \in \mathscr{B}_{r}\left(\mathbf{q}^{r}\right)$}

The first assumption is adopted in the interest of space: an example is reported in Fig.1. In particular, we refer to these systems as square systems. The second assumption allows defining the evolution of $\mathbf{q}(t)$ in Sec.III and IV and can be satisfied in the points generated by the global path planner, hence such an $r$ exists by continuity arguments. The last assumption is guaranteed if the planner properly selects the waypoints such that $\left\|\mathbf{q}^{p}\left(t_{\ell+1}\right)-\mathbf{q}^{p}\left(t_{\ell}\right)\right\|$ is smaller than $r$.

\section{LOCAL PLANNER: CONTINOUS-TIME}

In this section, we describe the local planner that generates the desired vehicles velocity in the scenario of continuous time measurements. The goal is to steer $\mathbf{q}(t)$ to $\mathbf{q}^{r}$. Assuming $\mathbf{A}_{\mathbf{q}(t)}$ full-rank, from (2) the evolution of $\mathbf{q}$ is ruled by

$$
\dot{\mathbf{q}}(t)=\mathbf{A}_{\mathbf{q}(t)}^{-1} \mathbf{u}(t) .
$$

A possible choice to define the desired vehicles velocities is:

$$
\mathbf{u}(t)=-\mathbf{A}_{\mathbf{q}(t)} \mathbf{K}\left(\mathbf{q}(t)-\mathbf{q}^{r}\right),
$$

where $\mathbf{K}$ is a gain matrix to be designed. With this choice, if $\mathbf{A}_{\mathbf{q}(t)}$ is invertible, we have that

$$
\dot{\mathbf{q}}=-\mathbf{K}\left(\mathbf{q}(t)-\mathbf{q}^{r}\right) .
$$

The feedback gain $\mathbf{K}$ needs to guarantee stability, but also to make the trajectory $\mathbf{q}(t)$ not to pass through a singularity. This is established in the next Proposition.

Proposition 1. Consider the system (7)-(8) and Ass. I. If $\mathbf{K}+\mathbf{K}^{\top}$ is strictly positive definite, then

1) for all $t \geq 0, \mathbf{q}(t) \in \mathscr{B}_{r}\left(\mathbf{q}^{r}\right)$

2) the trajectory $\mathbf{q}(t)$ converges exponentially fast to $\mathbf{q}^{r}$.

Proof. 1) Take the Lyapunov function $V(\mathbf{q})=\left\|\mathbf{q}-\mathbf{q}^{r}\right\|^{2}$. Then its time derivative is as follow

$$
\dot{V}(\mathbf{q})=-\left(\mathbf{q}-\mathbf{q}^{r}\right)^{\top}\left(\mathbf{K}+\mathbf{K}^{\top}\right)\left(\mathbf{q}-\mathbf{q}^{r}\right)<0, \quad \mathbf{q} \neq \mathbf{q}^{r},
$$

if $\mathbf{K}+\mathbf{K}^{\top}>0$, i.e., if the symmetric part of $\mathbf{K}$ is so. Hence $V(\mathbf{q})$ does not increase and therefore $\mathbf{q}(t) \in \mathscr{B}_{r}\left(\mathbf{q}^{r}\right) \quad \forall t \geq 0$. 2) The solution of (9) is $\mathbf{q}(t)=e^{-\mathbf{K} t} \mathbf{q}^{0}+\mathbf{q}^{r}$ that converges exponentially fast to $\mathbf{q}^{r}$ if $-\mathbf{K}$ is Hurwitz which is guaranteed by the hypothesis $\mathbf{K}+\mathbf{K}^{\top}>0$.

We conclude this section observing that, if we choose $\mathbf{K}$ to be block diagonal of the form $\mathbf{K}=\operatorname{diag}\left\{\mathbf{K}_{1}, \ldots, \mathbf{K}_{N}, \mathbf{K}_{L}\right\}$, where $\mathbf{K}_{i} \in \mathbf{R}^{m_{i} \times m_{i}}$ and $\mathbf{K}_{L} \in \mathbf{R}^{m_{L} \times m_{L}}$, then

$$
\mathbf{u}_{i}=\mathbf{A}_{\mathbf{q}_{i}}^{(i)} \mathbf{K}_{i}\left(\mathbf{q}_{i}(t)-\mathbf{q}_{i}^{r}\right)+\mathbf{A}_{\mathbf{q}_{L}}^{(i)} \mathbf{K}_{L}\left(\mathbf{q}_{L}(t)-\mathbf{q}_{L}^{r}\right),
$$

i.e., the desired velocity for the $i$-th robot does not depend on the other robots state, as defined in (5).

\section{Local Planner: Sampled Measurements}

We now consider the evolution of (7) under sampled dynamics, that is, we assume that $\mathbf{q}$ is measured on the time instants $h T, h=0,1,2, \ldots$ where $T$ is the sampling time. Furthermore, we assume that the vehicles reference velocity $\mathbf{u}(t)$ is kept constant within a time window $T$ using (8):

$$
\mathbf{u}(t)=\mathbf{u}_{h}=-\mathbf{A}_{\mathbf{q}_{h}} \mathbf{K}\left(\mathbf{q}_{h}-\mathbf{q}^{r}\right), \quad h T \leq t<(h+1) T,
$$

for $h=1,2, \ldots$, and $\mathbf{q}_{h}:=\mathbf{q}(h T)$. $\mathbf{K}$ can be chosen to satisfy Prop. 1 and s.t. $\mathbf{u}_{i}$ does not depend on $\mathbf{q}_{j}$ with $i \neq j$ :

$$
\mathbf{K}=k \mathbf{I}_{n}, \quad k \in \mathbb{R}_{>0},
$$

with $\mathbf{I}_{n} \in \mathbb{R}^{n \times n}$ the identity matrix. In this scenario, assuming $A_{\mathbf{q}(t)}$ non singular, the evolution of $\mathbf{q}(t)$ becomes:

$$
\dot{\mathbf{q}}(t)=-k A_{\mathbf{q}(t)}^{-1} A_{\mathbf{q}_{h}}\left(\mathbf{q}_{h}-\mathbf{q}^{r}\right), \quad h T \leq t<(h+1) T .
$$

The main goal of this section is to design $k$, possibly time varying, i.e., $k=k_{h}$, such that the stability of the system is still guaranteed. For the sake of notational convenience, we apply a change of coordinates of type $\mathbf{e}(t) \leftarrow \mathbf{q}(t)-\mathbf{q}^{r}$ and the simplified notation $\mathscr{B}_{0}:=\mathscr{B}_{r}\left(\mathbf{e}^{r}\right)$, with $\mathbf{e}^{r}=\mathbf{0}$.

The design of $k$ is based on the study of the following auxiliary system whose solution is characterized by interesting and useful properties that will be analyzed later on:

$$
\begin{aligned}
& \dot{\mathbf{e}}^{\prime}\left(\tau ; \mathbf{e}_{h}\right)=-A_{\mathbf{e}^{\prime}\left(\tau ; \mathbf{e}_{h}\right)}^{-1} A_{\mathbf{e}_{h}} \mathbf{e}_{h}=: \mathbf{f}\left(\mathbf{e}^{\prime}\left(\tau ; \mathbf{e}_{h}\right)\right) \\
& \mathbf{e}^{\prime}\left(0 ; \mathbf{e}_{h}\right)=\mathbf{e}_{h} ; \quad \mathbf{e}_{h} \in \mathscr{B}_{0},
\end{aligned}
$$

where $\tau \in[0, k T]$ and $\mathbf{e}^{\prime}(\cdot ; \cdot) \in \mathbb{R}^{m}$. By direct inspection

$$
\mathbf{e}(t)=\mathbf{e}^{\prime}\left(k(t-h T) ; \mathbf{e}_{h}\right), \quad h T \leq t<(h+1) T .
$$

Hence, once the solution $\mathbf{e}^{\prime}\left(\tau ; \mathbf{e}_{h}\right)$ is computed, then $\mathbf{e}(t)$ is obtained through shifting by $h T$ and rescaling by $k$ as long as $\mathbf{e}^{\prime}\left(k(t-h T) ; \mathbf{e}_{h}\right)$ exists; then, $\mathbf{q}(t)=\mathbf{e}(t)+\mathbf{q}^{r}$. The major benefit of this approach is that the analysis of (12) is independent of the gain $k$ and the sampling period $T$. From Ass. 1, and since $\mathbf{f}$ and $\frac{\partial \mathbf{f}}{\partial \mathbf{e}^{\prime}}$ are continuous maps on a compact domain, the following properties follow, for some $a, b>0$

$$
\begin{aligned}
\mathbf{f}\left(\mathbf{e}^{\prime}(0 ; \mathbf{e})\right) & =-\mathbf{e}, \quad \forall \mathbf{e} \in \overline{\mathscr{B}}_{0} \\
\left\|\mathbf{f}\left(\mathbf{e}^{\prime}(\tau) ; \mathbf{e}\right)\right\| & \leq\left\|A_{\mathbf{e}^{\prime}}^{-1} A_{\mathbf{e}}\right\|\|\mathbf{e}\|=a\|\mathbf{e}\|, \forall \mathbf{q}^{\prime}, \mathbf{e} \in \overline{\mathscr{B}}_{0}( \\
\left\|\frac{\partial \mathbf{f}\left(\mathbf{e}^{\prime}(\tau) ; \mathbf{e}\right)}{\partial \mathbf{e}^{\prime}}\right\| & \leq b\|\mathbf{e}\|, \quad \forall \mathbf{e}^{\prime}, \forall \mathbf{e} \in \overline{\mathscr{B}}_{0},
\end{aligned}
$$

Since the flow $\mathbf{f}\left(\mathbf{e}^{\prime} ; \mathbf{e}_{h}\right)$ is locally continuously differentiable in $\mathbf{e}^{\prime}$, then for each $\mathbf{e}_{h} \in \mathscr{B}_{0}$ there exists $\delta\left(\mathbf{e}_{h}\right)>0$ s.t. (12) has a unique solution $\mathbf{e}^{\prime}\left(\tau ; \mathbf{e}_{h}\right)$ for $\tau \in\left[0, \delta\left(\mathbf{e}_{h}\right)\right)$. Without loss of generality we define $\tau_{\max }\left(\mathbf{e}_{h}\right)$ the maximum time extension for which the unique solution $\mathbf{e}^{\prime}\left(\tau ; \mathbf{e}_{h}\right)$ exists for $\tau \in\left[0, \tau_{\max }\left(\mathbf{e}_{h}\right)\right)$. An interesting property of $\mathbf{e}^{\prime}\left(\tau ; \mathbf{e}_{h}\right)$ is described in Prop. 2:

Proposition 2. Consider the dynamical system (12) and assume Ass. 1 holds true. Then the solution $\mathbf{e}^{\prime}\left(\tau ; \mathbf{e}_{h}\right)$ satisfies one of these two properties:

(i) for all $\tau>0$, it holds $\left\|\mathbf{e}^{\prime}\left(\tau ; \mathbf{e}_{h}\right)\right\|<\left\|\mathbf{e}_{h}\right\|$ and in such case $\tau_{\max }\left(\mathbf{e}_{h}\right)=\infty$; 


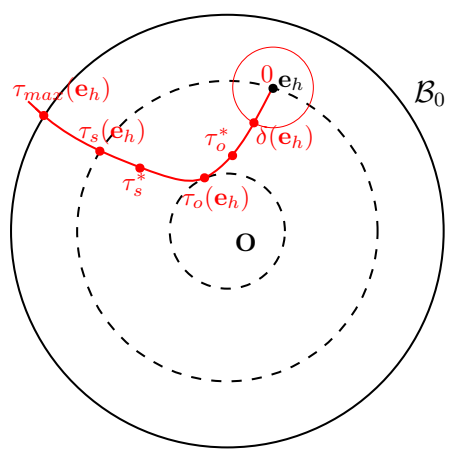

Fig. 3: Depiction of $\mathscr{B}_{0}, \delta_{\mathbf{e}_{h}}, \tau_{o}^{*}, \tau_{o}\left(\mathbf{e}_{h}\right), \tau_{s}^{*}, \tau_{s}\left(\mathbf{e}_{h}\right)$ and $\tau_{\max }\left(\mathbf{e}_{h}\right)$. The time values are in red, and the points or sets in $\mathbb{R}^{m}$ in black.

(ii) there exists $0<\bar{\tau}<\tau_{\max }\left(\mathbf{e}_{h}\right)$ such that $\left\|\mathbf{e}^{\prime}\left(\bar{\tau} ; \mathbf{e}_{h}\right)\right\|=$ $\left\|\mathbf{e}_{h}\right\|$ and $\left\|\mathbf{e}^{\prime}\left(\tau ; \mathbf{e}_{h}\right)\right\|<\left\|\mathbf{e}_{h}\right\|$ for all $0<\tau<\bar{\tau}$.

Proof. Only two scenarios are possible, either $\left\|\mathbf{e}^{\prime}\left(\tau ; \mathbf{e}_{h}\right)\right\|<$ $\left\|\mathbf{e}_{h}\right\|$ for all $\tau>0$, from which $\tau_{\max }\left(\mathbf{e}_{h}\right)=\infty$ follows, or not. If not, then $\bar{\tau}:=\inf _{\tau>0}\left\{\tau \mid\left\|\mathbf{e}^{\prime}\left(\tau ; \mathbf{e}_{h}\right)\right\| \geq\left\|\mathbf{e}_{h}\right\|\right\}$ is well defined and finite. Now, let us assume that $\bar{\tau}=0$ : since $\mathbf{e}^{\prime}\left(\tau ; \mathbf{e}_{h}\right)$ is continuously differentiable in $\tau$ and since $\dot{\mathbf{e}}^{\prime}\left(0 ; \mathbf{e}_{h}\right)=-\mathbf{e}_{h}$, then there exists $\dot{\mathbf{e}}^{\prime}\left(0 ; \mathbf{e}_{h}\right)$ s.t. $\mathbf{e}_{h}^{\top} \dot{\mathbf{e}}^{\prime}\left(0 ; \mathbf{e}_{h}\right) \geq 0 \Leftrightarrow-\mathbf{e}_{h}^{\top} \mathbf{e}_{h}=$ $-\left\|\mathbf{e}_{h}\right\|^{2} \leq 0$ that is a contradiction. This implies that $\bar{\tau}>0$. Finally, observe that the definition of $\bar{\tau}$ makes sense only if a solution $\mathbf{e}^{\prime}\left(\tau ; \mathbf{e}_{h}\right)$ exists, hence $\bar{\tau} \leq \tau_{\max }\left(\mathbf{e}_{h}\right)$.

Based on the previous result, we can now define the following temporal variables:

$$
\begin{aligned}
& \tau_{s}\left(\mathbf{e}_{h}\right):=\min _{\tau}\left\{\tau>0 \mid\left\|\mathbf{e}^{\prime}\left(\tau ; \mathbf{e}_{h}\right)\right\|=\left\|\mathbf{e}_{h}\right\|\right\}, \quad \tau_{s}^{*}:=\inf _{\mathbf{e}_{h} \in \mathscr{B}_{0}} \tau_{s}\left(\mathbf{e}_{h}\right), \\
& \tau_{o}\left(\mathbf{e}_{h}\right):=\underset{0 \leq \tau \leq \tau_{s}\left(\mathbf{e}_{h}\right)}{\operatorname{arginf}}\left\|\mathbf{e}^{\prime}\left(\tau ; \mathbf{e}_{h}\right)\right\|, \quad \tau_{o}^{*}:=\inf _{\mathbf{q}_{h} \in \mathscr{B}_{0}} \tau_{o}\left(\mathbf{q}_{h}\right),
\end{aligned}
$$

where $\tau_{s}\left(\mathbf{e}_{h}\right)=\infty$ if $\left\|\mathbf{e}^{\prime}\left(\tau ; \mathbf{e}_{h}\right)\right\|<\left\|\mathbf{e}_{h}\right\|, \forall \tau$. These quantities are sketched in Fig.3. Basically, $\tau_{s}\left(\mathbf{e}_{h}\right)$ represents the first time that the solution $\mathbf{e}^{\prime}\left(\tau ; \mathbf{e}_{h}\right)$ hits the boundary of the ball centered at the origin and passing through the initial condition $\mathbf{e}_{h}$, while $\tau_{o}\left(\mathbf{e}_{h}\right)$, represents the time that $\mathbf{e}^{\prime}\left(\tau ; \mathbf{e}_{h}\right)$ is closest to the origin. We will show in the next section, that for any given set $\mathscr{B}_{0}$ we can find $0<\bar{\tau}_{o}<\bar{\tau}_{s}$ and $0 \leq \rho<1$ s.t. $\left\|\mathbf{e}^{\prime}\left(\bar{\tau}_{o} ; \mathbf{e}_{h}\right)\right\| \leq \rho\left\|\mathbf{e}_{h}\right\|$ for all $\mathbf{e}_{h} \in \mathscr{B}_{0}$, and $\bar{\tau}_{o} \leq \tau_{o}^{*}, \bar{\tau}_{s} \leq \tau_{s}^{*}$. The variables $\bar{\tau}_{o}$ and $\tau_{o}\left(\mathbf{e}_{h}\right)$ allow proposing two different strategies to design the gain $k$. The first is based on the observation that if $k=\frac{\tau_{o}}{T}$, then $\mathbf{e}^{\prime}\left(\tau ; \mathbf{e}_{h}\right) \rightarrow$ $\mathbf{0} \forall \mathbf{e}_{h}$ at a convergence rate $\rho$. In fact, from (13) we have $\left\|\mathbf{e}_{h+1}\right\|=\left\|\mathbf{e}^{\prime}\left(k T ; \mathbf{e}_{h}\right)\right\|=\left\|\mathbf{e}^{\prime}\left(\bar{\tau}_{o} ; \mathbf{e}_{h}\right)\right\| \leq \rho\left\|\mathbf{e}_{h}\right\|$. This suggests an offline procedure to select $k$ that will be described in the next Sect. IV-A. However, based on the definition of $\tau_{o}\left(\mathbf{e}_{h}\right)$, it might be likely that $\left\|\mathbf{e}^{\prime}\left(\tau_{o}\left(\mathbf{e}_{h}\right) ; \mathbf{e}_{h}\right)\right\|<\left\|\mathbf{e}^{\prime}\left(\tau_{o}^{*} ; \mathbf{e}_{h}\right)\right\|$ for most $\mathbf{e}_{h} \in \mathscr{B}_{0}$. Therefore, an alternative approach is to select $k$ at each instant $h$ such that $k_{h}:=\frac{\tau_{o}\left(\mathbf{e}_{h}\right)}{T}$. This idea suggests an online strategy that will be described in Sect. IV-B.

\section{A. Off-line procedure (Stability and convergence rate)}

From (13), if we show that $\tau_{s}^{*}>0$, then the original system (12) is asymptotically stable for

$$
k T<\tau_{s}^{*} \quad \forall \mathbf{e}(0) \in \mathscr{B}_{0} .
$$

Note that if this condition is not satisfied, then we can find a time instant $\tau^{\prime} \in\left[\tau_{s}^{*},+\infty\right]$ and $\mathbf{e}(0)$ such that $\left\|\mathbf{e}^{\prime}\left(\tau^{\prime} ; \mathbf{e}(0)\right)\right\|>$ $\left\|\mathbf{e}^{\prime}(0 ; \mathbf{e}(0))\right\|$, that is $\|\mathbf{e}(k T)\|>\|\mathbf{e}(0)\|$ for $k=\frac{\tau^{\prime}}{T}$. Although this does not imply instability of the whole trajectory, it is an undesired behavior. We now want to find an explicit lower bound $\bar{\tau}_{s}>0$ for $\tau_{s}^{*}$. To do that, we consider an expansion of the solution of (12) and numerically estimate the upper bound of the approximation error via an additional parameter $\mu$. This allows deriving an analytical expression of $\bar{\tau}_{s}$. We recall that the solution of (12) can also be written as:

$$
\mathbf{e}^{\prime}\left(\tau ; \mathbf{e}_{h}\right)=\mathbf{e}_{h}+\int_{0}^{\tau} \mathbf{f}\left(\mathbf{e}^{\prime}\left(\tau^{\prime} ; \mathbf{e}_{h}\right)\right) d \tau^{\prime}, \quad 0 \leq \tau<\tau_{s}\left(\mathbf{e}_{h}\right) .
$$

By using Taylor's theorem for multivariate functions with integral form of the remainder, it becomes

$$
\begin{aligned}
\mathbf{e}^{\prime}\left(\tau ; \mathbf{e}_{h}\right) & =\mathbf{e}_{h}+\tau \mathbf{f}\left(\mathbf{e}^{\prime}(0) ; \mathbf{e}_{h}\right)+ \\
& +\tau^{2} \int_{0}^{1}(1-\varepsilon) \frac{\partial \mathbf{f}\left(\mathbf{e}^{\prime}(\varepsilon \tau) ; \mathbf{e}_{h}\right)}{\partial \mathbf{e}^{\prime}} \mathbf{f}\left(\mathbf{e}^{\prime}(\varepsilon \tau) ; \mathbf{e}_{h}\right) d \varepsilon \\
& =(1-\tau) \mathbf{e}_{h}+\tau^{2} \mathbf{d}\left(\tau, \mathbf{e}_{h}\right), \quad 0 \leq \tau<\tau_{s}\left(\mathbf{e}_{h}\right),
\end{aligned}
$$

where the reminder $\mathbf{d}$ has the property ${ }^{2}$ :

Proposition 3. There exists $0<\lambda<\infty$ such that for all $q_{h} \in$ $\mathscr{B}_{0}$ and for all $0<\tau<\tau_{s}\left(\mathbf{e}_{h}\right)$ it holds $\left\|\mathbf{d}\left(\tau, \mathbf{e}_{h}\right)\right\| \leq \lambda\left\|\mathbf{e}_{h}\right\|^{2}$.

Proof. As a consequence of the properties (15) and (16), the reminder in (17) becomes

$$
\begin{aligned}
\left\|\mathbf{d}\left(\tau, \mathbf{e}_{h}\right)\right\| & \leq \int_{0}^{1}(1-\varepsilon)\left\|\frac{\partial \mathbf{f}\left(\mathbf{e}^{\prime}(\varepsilon \tau) ; \mathbf{e}_{h}\right)}{\partial \mathbf{e}^{\prime}}\right\|\left\|\mathbf{f}\left(\mathbf{e}^{\prime}(\varepsilon \tau) ; \mathbf{e}_{h}\right)\right\| d \varepsilon \\
& \leq \int_{0}^{1}(1-\varepsilon) a b\left\|\mathbf{e}_{h}\right\|^{2} d \varepsilon=\frac{1}{2} a b\left\|\mathbf{e}_{h}\right\|^{2}=\lambda\left\|\mathbf{e}_{h}\right\|^{2}
\end{aligned}
$$

where $\lambda:=\frac{1}{2} a b$ and $a, b$ are the bounding constants introduced in (15) and (16).

Notice that since $\mathbf{e}_{h} \in \mathscr{B}_{o}$, then $\left\|\mathbf{e}_{h}\right\| \leq r$ and there exists

$$
\mu:=\frac{1}{2} a b r
$$

such that $\left\|\mathbf{d}\left(\tau, \mathbf{e}_{h}\right)\right\| \leq \frac{1}{2} a b\left\|\mathbf{e}_{h}\right\|^{2} \leq \mu\left\|\mathbf{e}_{h}\right\|$. Note that $\mu$ represents a rough estimate of the upper bound of $\left\|\mathbf{d}\left(\tau, \mathbf{e}_{h}\right)\right\|$. However, this estimate can be refined as follows. Let

$$
\begin{aligned}
\mathbf{d}^{\prime}\left(\tau, \mathbf{e}_{h}\right):= & \tau^{2} \mathbf{d}\left(\tau, \mathbf{e}_{h}\right) \stackrel{(17)}{=} \mathbf{e}^{\prime}\left(\tau, \mathbf{e}_{h}\right)-(1-\tau) \mathbf{e}_{h} \quad \text { and } \\
\mu^{*}:= & \inf _{\gamma}\left\{\gamma \mid\left\|\mathbf{d}^{\prime}\left(\tau, \mathbf{e}_{h}\right)\right\| \leq \gamma\left\|\mathbf{e}_{h}\right\| \tau^{2},\right. \\
& \left.\forall \mathbf{e}_{h} \in \mathscr{B}_{0}, \forall \tau \in\left(0, \tau_{s}\left(\mathbf{e}_{h}\right)\right)\right\} .
\end{aligned}
$$

We are interested in providing an estimate $\hat{\mu}^{*}$ of $\mu^{*}$. To this aim, we randomly pick samples in $\mathscr{B}_{0}, \mathbf{e}_{h}^{i} \in \mathscr{B}_{0}$, and for each of them we simulate $\mathbf{e}^{\prime}\left(\tau_{i} ; \mathbf{e}_{h}^{i}\right)$ for $\tau_{i} \in\left[0, \tau_{s}\left(\mathbf{e}_{h}^{i}\right)\right]$.

$$
\hat{\mu}^{*}:=\max _{i}\left\{\frac{\left\|\mathbf{d}^{\prime}\left(\tau_{i}, \mathbf{e}_{h}^{i}\right)\right\|}{\tau_{i}^{2}\left\|\mathbf{e}_{h}^{i}\right\|}, \forall \mathbf{e}_{h}^{i} \in \mathscr{B}_{0}, \forall \tau_{i} \in\left(0, \tau_{s}\left(\mathbf{e}_{h}^{i}\right)\right)\right\} .
$$

Since $\hat{\mu}$ is computed on a sampled $\mathscr{B}_{0}$, we may discard some configurations which would give a larger value of the

\footnotetext{
${ }^{2}$ Note that the reminder scales as $\left\|\mathbf{e}_{h}\right\|^{2}$ which implies that as $\mathbf{e}^{\prime}$ becomes closer to the origin, the faster it converges.
} 

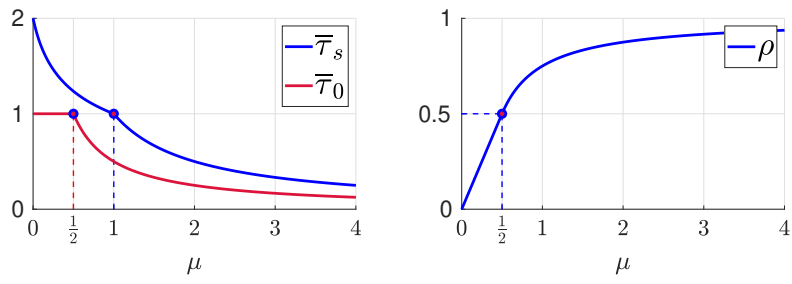

Fig. 4: Representation of $\bar{\tau}_{s}(\mu), \bar{\tau}_{0}(\mu)$ and $\rho(\mu)$.

estimate. Hence $\hat{\mu} \leq \mu$ and $\hat{\mu} \rightarrow \mu$ as the number of samples increases. From (17) and Prop. 3, for $\mathbf{e}_{h} \in \mathscr{B}_{0}$ we have that

$$
\left\|\mathbf{e}^{\prime}\left(\tau ; \mathbf{e}_{h}\right)\right\| \leq\left(|1-\tau|+\mu \tau^{2}\right)\left\|\mathbf{e}_{h}\right\|,
$$

for all $0 \leq \tau<\tau_{s}\left(\mathbf{e}_{h}\right)$. In order to evaluate upper bounds for the convergence rate, we need to study the following function

$$
g(\tau ; \mu):=|1-\tau|+\mu \tau^{2}
$$

We start our analysis with the following result.

Proposition 4. Given $\mu$, the function $g(\tau ; \mu)$ is strictly smaller than 1, i.e., $g(\tau ; \mu)<1$ for $\tau \in\left(0, \bar{\tau}_{s}(\mu)\right)$, where

$$
\bar{\tau}_{s}(\mu):=\left\{\begin{array}{cl}
\frac{4}{1+\sqrt{1+8 \mu}} & \text { if } \mu<1 \\
\frac{1}{\mu} & \text { if } \mu \geq 1
\end{array},\right.
$$

where $\bar{\tau}_{s}(\mu)$ is such that $g\left(\bar{\tau}_{s}(\mu) ; \mu\right)=1$.

The proof is available in Appendix A. Note that since $\bar{\tau}_{s}$ is obtained by using upper bounds on some terms, it holds that $\bar{\tau}_{s} \leq \tau_{s}^{*} \leq \tau_{\max }\left(\mathbf{e}_{h}\right)$. Hence, in the following set

$$
\mathscr{T}:=\{\tau \mid g(\tau ; \mu)<1\}=\left(0, \bar{\tau}_{s}(\mu)\right),
$$

the norm $\left\|\mathbf{e}^{\prime}\left(\tau ; \mathbf{e}_{h}\right)\right\|$ decreases w.r.t. $\left\|\mathbf{e}_{h}\right\|$. We now want to find the time $\bar{\tau}_{o}(\mu) \leq \tau_{o}^{*}$ in order to maximally decrease toward the origin, and the relative decreasing rate $\rho(\mu)$, i.e.,

$$
\bar{\tau}_{o}(\mu):=\arg \min _{\tau} g(\tau ; \mu), \quad \rho(\mu)=g\left(\bar{\tau}_{o}(\mu) ; \mu\right) .
$$

The following proposition provides the values attained by $\bar{\tau}_{o}(\mu)$ and $\rho(\mu)$, which are obtained from their definitions and the computation of $g(\tau ; \mu)$.

Proposition 5. Consider function $g(\tau ; \mu)$. Then

$\bar{\tau}_{o}(\mu)=\left\{\begin{array}{ll}1 & \mu<\frac{1}{2} \\ \frac{1}{2 \mu} & \mu \geq \frac{1}{2}\end{array} \quad\right.$ and $\rho(\mu)=\left\{\begin{array}{ll}\mu & \mu<\frac{1}{2} \\ 1-\frac{1}{4 \mu} & \mu \geq \frac{1}{2}\end{array}\right.$.

Note that $\rho<1$ as shown in Fig. 4. The proof is available Appendix A. A representation of $\bar{\tau}_{s}(\mu), \bar{\tau}_{o}(\mu)$ and $\rho(\mu)$ is reported in Fig. 4. Note that if $\mu=0$ then $\rho=0$ and we obtain a dead-beat controller. Indeed, from (20) with $k=\frac{\bar{\tau}_{o}}{T}$, it holds $\|\mathbf{e}(t)\|=\left\|\mathbf{e}^{\prime}\left(\bar{\tau}_{o}(\mu) ; \mathbf{e}_{h}\right)\right\|=0$ in one step. Moreover, for $\mu<\frac{1}{2}, \bar{\tau}_{o}(\mu)=1$ regardless of $\mu$ and this is an indication of robustness. The previous result suggests that a possible choice for the optimal offline gain $k$, once the sampling time $T$ is known, is

$$
k^{*}=\frac{\bar{\tau}_{o}(\mu)}{T},
$$

as formally established in the next proposition.
Proposition 6. For all $\mathbf{e}(0) \in \mathscr{B}_{0}$ the following inequality holds:

$$
\|\mathbf{e}(h T)\| \leq \rho^{h}(\mu)\|\mathbf{e}(0)\|
$$

and $\|\mathbf{e}(t)\| \leq\|\mathbf{e}(h T)\|$ for all $h T \leq t<(h+1) T$.

Proof. From Prop. 1 and (20) and recalling that $\rho(\mu)<1$ :

$$
\left\|\mathbf{e}_{h+1}\right\| \leq\left\|\mathbf{e}^{\prime}\left(\tau ; \mathbf{e}_{h}\right)\right\| \leq \rho(\mu)\left\|\mathbf{e}_{h}\right\| \leq g(\tau ; \mu)\left\|\mathbf{e}_{h}\right\|
$$

Hence $\left\|\mathbf{e}_{h+1}\right\| \leq \rho(\mu)\left\|\mathbf{e}_{h}\right\| \leq \rho(\mu)^{2}\left\|\mathbf{e}_{h-1}\right\| \leq \rho(\mu)^{h+1}\left\|\mathbf{e}_{0}\right\|$.

Notice that, from Prop. 6, it turns out that the origin is an asymptotically stable equilibrium for the system and the proposed offline strategy converges exponentially fast with a rate at least $\rho(\mu), \forall \mathbf{e}(0) \in \mathscr{B}_{0}$ that is included in the corresponding basin of attraction. We conclude this section observing that, since $\mu$ can be computed apriori before running the algorithm, then the offline strategy is amenable of both distributed and centralized implementations.

\section{B. Online model-predictive procedure}

In this section we consider the possibility to numerically compute the future trajectory $\mathbf{e}^{\prime}\left(\tau, \mathbf{e}_{h}\right)$ based on the model dynamics $\mathbf{f}\left(\mathbf{q} ; \mathbf{e}_{h}\right)$ and the current position $\mathbf{e}_{h}$. This implies that also $\tau_{o}\left(\mathbf{e}_{h}\right)$ can be computed at any time step $h$. If so, under the assumption that the input is kept constant for the following time interval $T$, we can propose the following input

$$
\mathbf{u}(t)=\mathbf{u}_{h}=-k_{h} A_{\mathbf{e}_{h}} \mathbf{e}_{h}, \quad h T \leq t<(h+1) T,
$$

where

$$
k_{h}:=\frac{\tau_{o}\left(\mathbf{e}_{h}\right)}{T} .
$$

A more precise characterization of the convergence properties of this strategy is stated in the next proposition.

Proposition 7. Consider the system in (11) with a time varying sequence of gains $k_{0}, k_{1}, k_{2}, \ldots$, where the generic $k_{h}$ is given as in (23). Then the system satisfies the following properties:

- $\|\mathbf{e}(t)\| \leq\|\mathbf{e}(h T)\|$ for all $h T \leq t<(h+1) T$;

- the convergence rate of the sampled dynamics is at least quadratic;

- the gain $k_{h}$ tends to $\frac{1}{T}$ as $h \rightarrow \infty$, that is $\lim _{h \rightarrow \infty} k_{h}=\frac{1}{T}$.

Proof. Observe that, according to (23), we necessarily have:

$$
\left\|\mathbf{e}_{h+1}\right\|=\left\|\mathbf{e}^{\prime}\left(\tau_{o}\left(\mathbf{e}_{h}\right) ; \mathbf{e}_{h}\right)\right\| \leq\left\|\mathbf{e}^{\prime}\left(\bar{\tau}_{o} ; \mathbf{e}_{h}\right)\right\| \leq \rho\left\|\mathbf{e}_{h}\right\|,
$$

hence the proposed scheme is exponentially stable with rate $\rho$ for any $T$. Since in the online scenario $r=\left\|\mathbf{e}_{h}\right\| \rightarrow 0$, then $\mu=\frac{1}{2} a b\left\|\mathbf{e}_{h}\right\| \rightarrow 0$. As so, there exists $\bar{h}$ s.t. $\mu<\frac{1}{2}$ for $h>\bar{h}$. Then from Prop. 5, $\rho(\mu)=\mu$ and $\left\|\mathbf{e}_{h+1}\right\| \leq \rho\left\|\mathbf{e}_{h}\right\|=$ $\frac{1}{2} a b\left\|\mathbf{e}_{h}\right\|^{2}$ for $h>\bar{h}$. As a consequence

$$
\limsup _{h \rightarrow+\infty} \frac{\left\|\mathbf{e}_{h+1}\right\|}{\left\|\mathbf{e}_{h}\right\|^{2}} \leq \frac{1}{2} a b,
$$

and $\left\|\mathbf{e}_{h}\right\| \leq\left(\frac{1}{2} a b\left\|\mathbf{e}_{0}\right\|\right)^{\left(2^{h}-1\right)}\left\|\mathbf{e}_{0}\right\|$. Since $a b>0$, then the quadratic convergence of the sequence $\left\|\mathbf{e}_{h}\right\|$ is guaranteed. Moreover, $\mu \rightarrow 0$ implies $\left\|\mathbf{d}\left(\tau, \mathbf{e}_{h}\right)\right\| \rightarrow 0$ and the second 


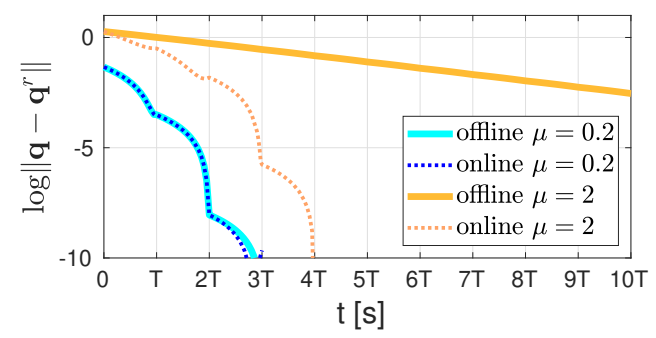

Fig. 5: In the figure, label offline refers to the choice (22), while label online refers to (23).

term in (17) becomes negligible. So, $\mathbf{e}^{\prime}\left(\tau ; \mathbf{e}_{h}\right) \rightarrow(1-\tau) \mathbf{e}_{h}$ is minimized for $\tau=1$. In the online scenario, the optimal gain is chosen as in (23), where $\tau_{o}\left(\mathbf{e}_{h}\right)$ corresponds to the minimum norm, hence $\tau_{o}\left(\mathbf{e}_{h}\right)=1$ and $k_{h}=\frac{1}{T}$.

Based on the definition of $\tau_{o}\left(\mathbf{e}_{h}\right)$ and on Prop. 7, we expect the online strategy to exhibit a faster convergence than the offline one. This fact is supported also by the numerical results reported in the next section. However, the higher rate of convergence comes at the price of a heavier computational load. Indeed $\tau_{o}\left(\mathbf{e}_{h}\right)$ needs to be estimated at each iteration and a global knowledge of the vector $\mathbf{e}_{h}$ is required; this implies that the online strategy cannot be implemented distributively, but only in a centralized fashion.

\section{Simulation Results}

The performance of the proposed steering method is evaluated and compared in this section, reporting the results obtained in Matlab. The platform edges of the simulated system (the one in Fig. 1) are $1[\mathrm{~m}]$ long and the cables linking the robots to the load measure $1.5[\mathrm{~m}]$. The sampling time is $T=0.01[\mathrm{~s}]$. The quantity $\hat{\mu}^{*}$ is estimated both for a small $\mathscr{B}_{o}$ with radius $r_{1}=0.08$ (that gave $\hat{\mu}^{*}<\frac{1}{2}$ ) and for a larger one with $r_{2}=0.5\left(\hat{\mu}^{*}>\frac{1}{2}\right)$, following the reasoning of Sec. IV-A. Observe that $r$ could be set as an optimization parameter to generate the reference path.

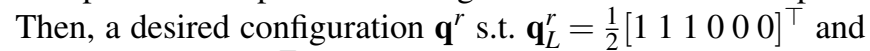
$\mathbf{q}_{\alpha}^{r}=\left[75^{\circ} 75^{\circ} 75^{\circ}\right]^{\top}$ is chosen and two initial conditions are considered, one s.t. $\left\|\mathbf{q}_{1}(0)-\mathbf{q}^{r}\right\|<r_{1}$ and the second s.t. $r_{1}<\left\|\mathbf{q}_{2}(0)-\mathbf{q}^{r}\right\|<r_{2}$. Finally, from Prop. 5, $\bar{\tau}_{o}$ is obtained based on the estimate $\hat{\mu}^{*}$, while the gain $k^{*}$ is computed with (22). At this point, the system in (12) is simulated until $\tau=\bar{\tau}_{o}$ using the Matlab function ODE; then, the solution of the original system, for $h T \leq t<(h+1) T$, is retrieved using (13). The procedure is repeated for every iteration. Conversely, in the online strategy the optimal gain $k=k_{h}$ that brings the system the closest to the desired configuration, is recomputed at each instant $h T$ based on the time $\tau_{o}\left(\mathbf{e}_{h}\right)$. We implicitly assumed that the machine used for the simulations can compute $k_{h}$ in less than $T$ seconds. Moreover, $T$ can be designed by the path planner to satisfy this condition. Fig. 5 shows the simulation results described above, where, for $\mu$ smaller and greater than $\frac{1}{2}$, the online and offline strategies are compared. We observe that for $\mu<\frac{1}{2}$ the two strategies have quite the same behavior, as expected from the proof of Prop. 6. Instead, as $\mu>\frac{1}{2}$, the offline strategy is not as efficient as the online one, since the convergence rate $\rho(\mu) \rightarrow 1$, as described in Prop. 5. In particular, it is emphasized the difference between the exponential (offline strategy) and doubly exponential (online) convergence rate described respectively in Prop. 6 and 7. The soundness of the proposed strategy is confirmed by preliminary simulations on a dynamic simulator with force controller, not reported here for space limitation.

\section{CONCLUSIONS}

In this work, we faced the problem of steering a multiagent manipulation system to a desired configuration by means of a feedback-based planner that exploits the sparse structure of the system and provides the desired vehicles velocities to be tracked by the system. We provided an extension from the continuous time case to the one with sampled measurements. This deals with a realistic scenario where wireless communication is employed. Finally, two different strategies were compared for the sampled scenario: the offline strategy converges exponentially fast and exploits a static control gain, while the second method is even faster, but computationally demanding since the gain must be computed online. Furthermore, if the second technique can be implemented only in a centralized fashion, the first one can be fulfilled also in a distributed way. Anyway, both the techniques do not require any communication among the robots. Some adaptations have been left for future work: we aim at studying the non-square systems $(n \neq m)$, analyzing them under different norms definitions. As discussed in Sec. II-B, there is potential for improving the trajectory generation, taking into account additional parameters as the inter-sample interval. Finally, we will apply the proposed strategy to a dynamical system and include a more realistic communication environment to simulate the whole structure.

\section{APPENDIX}

\section{A. Stability and Convergence analysis}

In this section we give the definition of the stability time $\bar{\tau}_{s}(\mu)$, the optimal time $\bar{\tau}_{o}(\mu)$ and the convergence rate $\rho(\mu)$. In order to evaluate upper bounds for asymptotic stability and rate of convergence, we need to study the following function

$g(\tau ; \mu):=|1-\tau|+\mu \tau^{2}= \begin{cases}1-\tau+\mu \tau^{2}=: g^{-}(\tau ; \mu) & \tau<1 \\ -1+\tau+\mu \tau^{2}=: g^{+}(\tau ; \mu) & \tau \geq 1\end{cases}$

We will study the function $g(\tau ; \mu)$ in three different scenarios: $\mu \in\left[0, \frac{1}{2}\right), \mu \in\left[\frac{1}{2}, 1\right)$ and $\mu \geq 1$. We start by observing that

$$
g(0 ; \mu)=1, g(1 ; \mu)=\mu, \frac{d g^{+}}{d \mu}=1+2 \mu \tau>0
$$

and by defining the minimum of $g^{-}(\tau ; \mu)$ and its minimizer w.r.t. $\tau$ as

$\tau_{p}(\mu)=\arg \min _{\tau} g^{-}(\tau ; \mu) \Leftrightarrow \frac{d g^{-}(\tau ; \mu)}{d \tau}=0 \Longrightarrow \tau_{p}(\mu)=\frac{1}{2 \mu}$ 
We now note that in the first scenario $\mu \in\left[0, \frac{1}{2}\right), \tau_{p}(\mu) \geq 1$ which implies that the function $g(\tau ; \mu)$ is monotonically decreasing for $\tau \in[0,1]$ and monotonically increasing for $\tau>1$.

In the second scenario $\mu \in\left[\frac{1}{2}, 1\right), \tau_{p}(\mu)<1$, therefore $g(\tau ; \mu)$ is monotonically decreasing for $\tau \in\left[0, \tau_{p}(\mu)\right]$ and monotonically increasing for $\tau>\tau_{p}(\mu)$.

Finally note that for $\mu<1, g(1 ; \tau)<1$, therefore there exists a unique $\bar{\tau}_{s}(\mu)$ such that $g\left(\bar{\tau}_{s}(\mu) ; \mu\right)=g^{+}\left(\bar{\tau}_{s}(\mu) ; \mu\right)=1$, while for $\mu>1, g(1 ; \tau)>1$, therefore there exists a unique $\bar{\tau}_{s}(\mu)$ such that $g\left(\bar{\tau}_{s}(\mu) ; \mu\right)=g^{-}\left(\bar{\tau}_{s}(\mu) ; \mu\right)=1$. A pictorial representation of the three scenarios is shown in Fig. 6. We are now ready to compute the stability region and convergence rate.

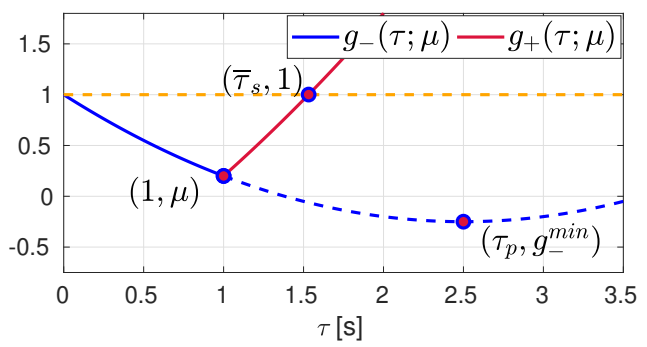

(a) $\mu \leq \frac{1}{2}$

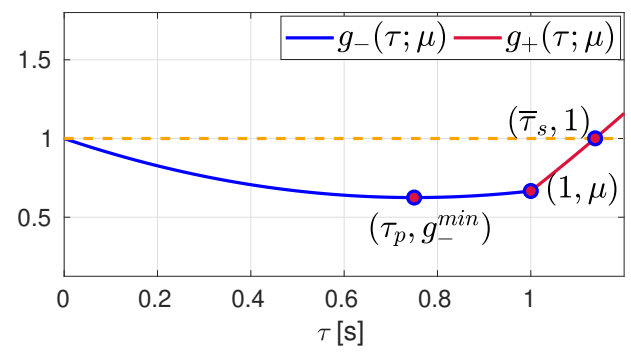

(b) $\frac{1}{2} \leq \mu \leq 1$

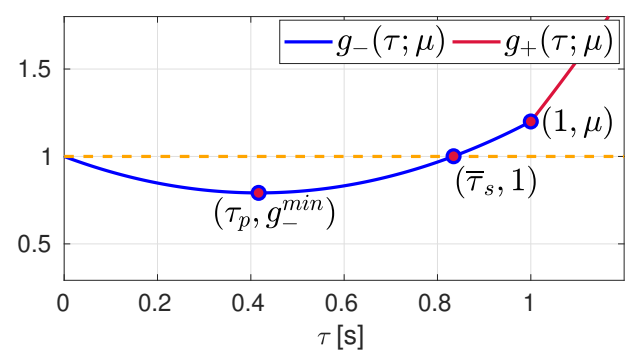

(c) $\mu \geq 1$

Fig. 6: Representation of $g(\tau ; \mu)$ in the three scenarios.

1) Stability $(g(\tau ; \mu)<1)$ : According to the analysis above, the stability set is given by:

$$
\mathscr{T}:=\{\tau \mid g(\tau ; \mu)<1\}=\left(0, \bar{\tau}_{s}(\mu)\right)
$$

More specifically, we have two scenarios depending whether the parameter $\mu$ is smaller or grater than unity.
If $\mu<1$ then $-1+\tau+\mu \tau^{2}=1$. Hence:

$$
\bar{\tau}_{s}(\mu)=\frac{-1+\sqrt{1+8 \mu}}{2 \mu}=\frac{4}{1+\sqrt{1+8 \mu}}
$$

while

$$
\mu>1 \Longrightarrow 1-\tau+\mu \tau^{2}=1 \Longrightarrow \bar{\tau}_{s}(\mu)=\frac{1}{\mu}
$$

which can be summarized in

$$
\bar{\tau}_{s}(\mu) \begin{cases}\frac{4}{1+\sqrt{1+8 \mu}} & \mu<1 \\ \frac{1}{\mu} & \mu \geq 1\end{cases}
$$

2) Optimal gain and rate $\left(\min _{\tau} g(\tau ; \mu)\right)$ : We now want to find the optimal stopping time $\bar{\tau}_{o}(\mu)$ in order to maximally decrease toward the origin, and the relative decrease rate $\rho(\mu)$, i.e.

$$
\bar{\tau}_{o}(\mu):=\arg \min _{\tau} g(\tau ; \mu), \quad \rho(\mu)=g\left(\bar{\tau}_{o}(\mu) ; \mu\right)
$$

Once again, we can distinguish two scenarios, depending whether the parameter $\mu$ is smaller or greater than $\frac{1}{2}$ More specifically, for $\mu<\frac{1}{2}$ the function $g^{-}(\tau ; \mu)$ is monotonically decreasing for $\tau<1$, and therefore $\tau_{o}(\mu)=1$, while for $\mu>\frac{1}{2}$ then $\tau_{o}(\mu)=\tau_{p}(\mu)=\frac{1}{2 \mu}$. This can be summarized as

$$
\bar{\tau}_{o}(\mu)= \begin{cases}1 & \mu<\frac{1}{2} \\ \frac{1}{2 \mu} & \mu \geq \frac{1}{2}\end{cases}
$$

By substitution is easy to verify that

$$
\rho(\mu)= \begin{cases}\mu & \mu<\frac{1}{2} \\ 1-\frac{1}{4 \mu} & \mu \geq \frac{1}{2}\end{cases}
$$

\section{REFERENCES}

[1] I. Maza, K. Kondak, M. Bernard, and A. Ollero, "Multi-UAV cooperation and control for load transportation and deployment," Journal of Intelligent \& Robotics Systems, vol. 57, no. 1-4, pp. 417-449, 2010.

[2] N. Michael, J. Fink, and V. Kumar, "Cooperative manipulation and transportation with aerial robots," Autonomous Robots, vol. 30, no. 1, pp. 73-86, Jan 2011.

[3] K. Sreenath and V. Kumar, "Dynamics, control and planning for cooperative manipulation of payloads suspended by cables from multiple quadrotor robots," in Robotics: Science and Systems, Berlin, Germany, June 2013.

[4] C. Masone, H. H. Blthoff, and P. Stegagno, "Cooperative transportation of a payload using quadrotors: A reconfigurable cable-driven parallel robot," in 2016 IEEE/RSJ International Conference on Intelligent Robots and Systems (IROS), Oct 2016, pp. 1623-1630.

[5] M. Manubens, D. Devaurs, L. Ros, and J. Cortés, "Motion planning for 6-D manipulation with aerial towed-cable systems," in 2013 Robotics: Science and Systems, Berlin, Germany, May 2013.

[6] Z. Wang and M. Schwager, "Multi-robot manipulation without communication," in Distributed Autonomous Robotic Systems, N.-Y. Chong and Y.-J. Cho, Eds. Tokyo: Springer Japan, 2016, pp. 135-149.

[7] H. Farivarnejad and S. Berman, "Stability and convergence analysis of a decentralized proportional-integral control strategy for collective transport," in 2018 Annual American Control Conference (ACC), June 2018, pp. 2794-2801.

[8] Y. Lim, S. Kwon, K. Kim, and H. Ahn, "Implementation of load transportation using multiple quadcopters," in 2017 IEEE International Conference on Advanced Intelligent Mechatronics (AIM), July 2017, pp. 639-644.

[9] M. Tognon, C. Gabellieri, L. Pallottino, and A. Franchi, "Aerial comanipulation with cables: The role of internal force for equilibria, stability, and passivity," IEEE Robotics and Automation Letters, Special Issue on Aerial Manipulation, vol. 3, no. 3, pp. 2577 - 2583, 2018. 
[10] A. Tagliabue, M. Kamel, R. Siegwart, and J. I. Nieto, "Robust collaborative object transportation using multiple mavs," CoRR, vol. abs/1711.08753, 2017.

[11] A. Tsiamis, C. K. Verginis, C. P. Bechlioulis, and K. J. Kyriakopoulos, "Cooperative manipulation exploiting only implicit communication," in 2015 IEEE/RSJ International Conference on Intelligent Robots and Systems (IROS), Sep. 2015, pp. 864-869.

[12] E. Rossi, M. Tognon, R. Carli, L. J. Cortés, and A. Franchi, "Discrete-time control of parallel kinematic systems," Univ. of Padova, Tech. Rep., Mar 2019. [Online]. Available: http://automatica. dei.unipd.it/tl_files/utenti2/rossi/tech_report.pdf 